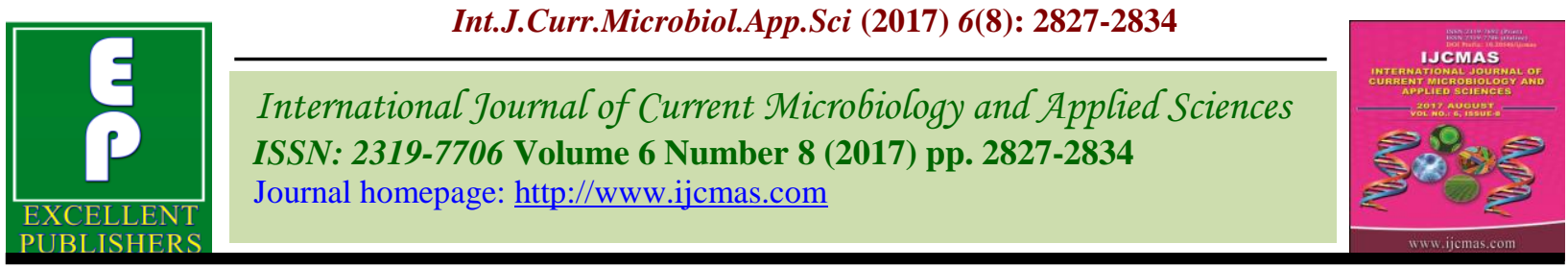

Original Research Article

https://doi.org/10.20546/ijcmas.2017.608.337

\title{
Combining Ability for Grain Yield and Its Components Different Environments in Wheat
}

\author{
Sunil Kumar Jatav ${ }^{1 *}$, B.R. Baraiya ${ }^{2}$ and V.S. Kandalkar ${ }^{1}$ \\ ${ }^{1}$ Department of Genetics and Plant Breeding, College of Agriculture, Gwalior, Rajmata \\ Vijayaraje Scindia Krishi Vishva Vidyalaya, Gwalior 474001, M.P., India \\ ${ }^{2}$ B.M. college of Agriculture, Khandwa, M.P., India \\ *Corresponding author
}

A B S T R A C T

Keywords

Bread wheat,

Combining

ability, sca, Gene

action, gca.

Article Info

Accepted:

23 June 2017

Available Online:

10 August 2017
Combining ability analysis was done in Line X Tester mating design by using 7 lines, 3 testers and $21 \mathrm{~F}_{1}$, $\mathrm{s}$ in a randomized block design with two replications during 2013-14 in sowing under water stress and non-stress environments. The analysis of genetic components revealed that both additive as well as non-additive components were prevalent for the control of grain yield and its components under both water stress and non-stress environments except spike length in under stress environment. The combining ability effects revealed that parent DL 803-3 was identified as good general combiners for grain yield under both water stress and non-stress environments and GW190 and GW 173 for grain yield under stress environment. The significant specific good combing cross ' $G W$ 322 X GW 173' having one promising parent for grain yield under stress condition would be advanced through simple / recurrent selection in segregating generations. Most of the good specific combinations for various traits involved parents with high $\mathrm{X}$ low or low $\mathrm{X}$ low or low X high GCA effects. These genotypes may be used in breeding programs targeting high potential under drought stress. These parents may be used for varietal improvement through the simple / recurrent selection in segregating generations to increase in yield potential of wheat. This may lead in the fixation of both additive and nonadditive components while making improvement in grain yield and its attributes.

\section{Introduction}

Wheat (Triticum aestivum L.) is the second most important cereal crop after rice in the context to its antiquity and its use as source of food and energy in India. Wheat crop has occupied almost 29.9 million hectares and producing 95.85 million tones in India (Anonymous, 2016). Improvement in wheat production can be achieved by enhancing through the development of new cultivars having wider genetic base and better performance. Earlier research review revealed that both general and specific combining abilities were involved in the inheritance of grain yield and its components (Singh et al., 2000; Murliya and Sastry, 2001). Selection of parents together with information on nature and magnitude of gene action controlling grain yield and its attributing characters is prerequisites while improving the plant type efficiently.

Hence an attempt has been made to study suitable parents through general combining ability and specific combining ability for improvement in yield potential under stress and non-stress conditions. 


\section{Materials and Methods}

An experiment consisting $21 \mathrm{~F}_{1} \mathrm{~s}$ and their 10 parents was conducted along with 2 checks viz., MP-4010 and RVW-4106 in randomized block design with two replications during winter season 2013-14. Twenty one crosses were developed in Line $\mathrm{x}$ Tester mating design using 7 lines viz., C-306, DL 803-3, GW-190, GW-322, PBW-175, MACS 6222, HI 1544 and 3 tester's viz., HD-2932, GW173 and PBW-343.

The sowing was done by dibbling seeds in rows with spacing of $20 \mathrm{~cm}$ apart and $4-6 \mathrm{~cm}$ within row under water stress and non-stress environments. The recommended packages of practices were adopted for optimum crop growth. Observations were recorded on five randomly selected plants in each line for grain yield and its attributes (Table 1). Combining ability analysis was worked out in Line $\mathrm{X}$ Tester mating design by following Kempthorne (1957).

\section{Results and Discussion}

\section{Combining ability variances}

The mean sum of square due to genotypes in the non-stress condition, lines were significant only for biological yield and grain yield whereas testers were significant for days to maturity, plant height, spike length and grain filling period. But in stress condition, lines were significant for grain yield per plant, grain per spike and test weight and testers for grain yield per plant, days to heading, days to flowering, grain weight per spike, grain per spike, flag leaf area and biological yield. Crosses were significant for grain yield per plant, flag leaf area and biological yield in non-stress and days to heading and test weight in stress condition (Table 1,2). The similar reports were also published earlier by Drikvand et al., (2005) and Dhadhal et al.,
(2008) for seeds per spike, tillers per plant and grain yield per plant under both water stress and non-stress conditions.

General combining ability (GCA) or specific combining ability (SCA) variances were substantial for most of the characters, thereby, indicating impotence of both additive as well as non-additive components of genetic variance in the control of these traits. These results are in agreement with reported earlier on GCA and SCA variances for yield and yield components in wheat (Mahmood and Chowdhry, 2000). The additive components of variances were higher compared to dominance components of variances for all characters studied under both stress and nonstress environments. The ratio of genetic components " $\sigma^{2}$ gca / $\sigma^{2}$ sca" also showed more than one, indicating predominance of additive variances for almost all characters under both water stress and non-stress environments. The similar reports were also published earlier by Vanpariya et al., (2006) for days to heading, plant height, spike length and spikelet per spike. Yadav and Behl (2002) for days to flowering, plant height, tillers per plant, grain per spike, flag leaf area, test weight and grain yield per plant.

\section{General combining ability effects in under stress and non-stress condition}

The significant estimates of significant positive GCA effects revealed that 'DL 8033 ' was good general combiner for grain yield per plant; C 306 for harvest index; GW 190 for plant height; HD 2932 for spike length in non-stress situation (Table 3). However, genotype PBW 343 was bad general combiner as showed longer days to maturity and grain filling period. Present results are in agreement with those of Vanpariya et al., (2006) for days to heading, plant height, spike length, spikelet per spike under non-stress condition; Ali and Khan (1998) for grain yield per plant. 
Table.1 Analysis of Variance for combining ability for grain yield and yield contributing characters in wheat

\begin{tabular}{|c|c|c|c|c|c|c|c|c|c|c|c|c|c|c|c|}
\hline Sources & DF & \multicolumn{2}{|c|}{ Days to heading } & \multicolumn{2}{|c|}{ Days to flowering } & \multicolumn{2}{|c|}{ Days to maturity } & \multicolumn{2}{|c|}{ Grain filling period } & \multicolumn{2}{|c|}{ Plant height } & \multicolumn{2}{|c|}{ Tiller/plant } & \multicolumn{2}{|c|}{ Spike length } \\
\hline & & $\mathrm{S}$ & NS & $\mathrm{S}$ & NS & $\mathrm{S}$ & NS & $\mathrm{S}$ & NS & $\mathrm{S}$ & NS & $\mathrm{S}$ & NS & $\mathrm{S}$ & NS \\
\hline Lines & 6 & 10.13 & 4.66 & 8.97 & 4.11 & 1.76 & 1.67 & 11.05 & 7.39 & 96.00 & 82.19 & 5.07 & 6.63 & 3.98 & 0.67 \\
\hline Tester & 2 & $140.21 * *$ & 9.93 & $158.79 * *$ & 3.17 & 2.21 & $30.31 * *$ & $129.07 * *$ & $37.50 * *$ & 29.45 & $297.61 * *$ & 11.00 & 17.16 & 1.42 & $2.68 * *$ \\
\hline $\mathrm{LX} \mathrm{T}$ & 12 & 5.99 & 5.79 & 7.98 & 6.19 & 1.30 & 4.06 & 8.07 & 9.06 & 83.66 & 18.26 & 5.62 & 9.40 & 1.15 & 1.37 \\
\hline Error & 32 & 9.07 & 4.69 & 14.72 & 3.80 & 5.28 & 4.06 & 15.75 & 7.25 & 51.64 & 64.56 & 4.94 & 12.74 & 1.73 & 1.03 \\
\hline
\end{tabular}

Conti.............

\begin{tabular}{|c|c|c|c|c|c|c|c|c|c|c|c|c|c|c|c|}
\hline Sources & $\begin{array}{l}\mathrm{D} \\
\mathrm{F}\end{array}$ & \multicolumn{2}{|c|}{$\begin{array}{c}\text { Grain weight per } \\
\text { spike }\end{array}$} & \multicolumn{2}{|c|}{ Grain per spike } & \multicolumn{2}{|c|}{ Test weight } & \multicolumn{2}{|c|}{$\begin{array}{c}\text { Grain yield per } \\
\text { plant }\end{array}$} & \multicolumn{2}{|c|}{ Flag leaf area } & \multicolumn{2}{|c|}{ Harvest Index } & \multicolumn{2}{|c|}{ Biological Yield } \\
\hline & & $\mathrm{S}$ & NS & $\mathrm{S}$ & NS & $\mathrm{S}$ & NS & $\mathrm{S}$ & NS & $\mathrm{S}$ & NS & $\mathrm{S}$ & NS & $\mathrm{S}$ & NS \\
\hline Lines & 6 & 0.12 & 0.27 & $\begin{array}{r}160.22 \\
*\end{array}$ & 70.22 & $85.94 * *$ & 14.19 & $19.62 * *$ & $32.29 * *$ & 173.54 & 65.41 & 39.40 & 225.53 & 68.96 & $186.29 * *$ \\
\hline Tester & 2 & $1.04 * *$ & 0.12 & $\begin{array}{r}382.24 \\
* *\end{array}$ & 16.88 & 15.99 & 21.74 & $51.68 * *$ & 10.85 & $438.01 * *$ & 83.51 & 745.51 & 134.91 & $\begin{array}{r}272.40 * \\
*\end{array}$ & 106.16 \\
\hline $\mathrm{LX} \mathrm{T}$ & 12 & 0.12 & 0.26 & 80.56 & 95.47 & $53.71 * *$ & 29.07 & $28.25 * *$ & $27.17 * *$ & 48.80 & $153.87 * *$ & 277.02 & 231.68 & $\begin{array}{r}103.51 * \\
*\end{array}$ & $237.90 * *$ \\
\hline Error & 32 & 0.23 & 0.37 & 79.69 & 117.01 & 23.50 & 29.21 & 4.89 & 9.93 & 99.97 & 67.12 & 293.64 & 232.33 & 28.04 & 54.34 \\
\hline
\end{tabular}

*, ** significant at 5 and 1 percent levels, respectively,

Table. 2 Genetic components estimates for 14 characters in wheat crosses in line $\mathrm{X}$ tester mating design

\begin{tabular}{|c|c|c|c|c|c|c|c|c|c|c|c|c|c|c|}
\hline \multirow[t]{2}{*}{ Covariance } & \multicolumn{2}{|c|}{ Days to heading } & \multicolumn{2}{|c|}{ Days to flowering } & \multicolumn{2}{|c|}{ Days to maturity } & \multicolumn{2}{|c|}{ Grain filling period } & \multicolumn{2}{|c|}{ Plant height } & \multicolumn{2}{|c|}{ Tiller/plant } & \multicolumn{2}{|c|}{ Spike length } \\
\hline & $\mathrm{S}$ & NS & $\mathrm{S}$ & NS & $S$ & NS & $\mathrm{S}$ & NS & $\mathrm{S}$ & NS & $\mathrm{S}$ & NS & $S$ & NS \\
\hline Cov HS (Line) & 0.690 & -0.188 & 0.165 & -0.347 & 0.077 & -0.399 & 0.496 & -0.278 & 2.057 & 10.655 & -0.092 & -0.461 & 0.471 & -0.117 \\
\hline Cov HS (Tester) & 9.587 & 0.296 & 10.772 & -0.216 & 0.065 & 1.875 & 8.643 & 2.032 & -3.872 & 19.954 & 0.384 & 0.555 & 0.019 & 0.093 \\
\hline Cov HS (Average) & -5.992 & -5.790 & -7.980 & -6.194 & -1.298 & -4.060 & -8.071 & -9.056 & -83.661 & -18.261 & -5.619 & -9.395 & -1.153 & -1.371 \\
\hline Cov FS & 29.510 & 8.772 & 32.570 & 8.606 & -0.031 & 9.389 & 27.587 & 17.441 & 120.580 & 58.411 & 8.635 & 11.686 & 1.765 & 2.099 \\
\hline \multicolumn{15}{|c|}{ Genetic components } \\
\hline$\sigma 2$ gca (Lines) & 0.690 & -0.188 & 0.165 & -0.347 & 0.077 & -0.399 & 0.496 & -0.278 & 2.057 & 10.655 & -0.092 & -0.461 & 0.471 & -0.117 \\
\hline$\sigma 2 \mathrm{gca}($ Testers $)$ & 2.762 & -0.754 & 0.659 & -1.389 & 0.310 & -1.595 & 1.984 & -1.111 & 8.228 & 42.622 & -0.369 & -1.843 & 1.883 & -0.467 \\
\hline$\sigma 2 \mathrm{gca}$ (Parents) & 876.58 & 25.77 & 981.22 & -21.76 & 6.42 & 168.23 & 789.47 & 183.22 & -340.00 & 1879.71 & 34.426 & 47.71 & 4.57 & 7.79 \\
\hline$\sigma 2 \mathrm{sca}$ & -1.540 & -11.57 & -15.96 & -12.38 & -2.595 & -8.11 & -16.143 & -18.11 & -167.32 & -36.52 & -11.23 & -18.79 & -2.30 & -2.74 \\
\hline$\sigma 2 \mathrm{gca} / \sigma 2 \mathrm{sca}$ & -569.05 & -2.23 & -61.48 & 1.76 & -2.47 & -20.72 & -48.91 & -10.12 & 2.03 & -51.47 & -3.06 & -2.54 & -1.98 & -2.84 \\
\hline$\sigma 2 \mathrm{~A}$ & 1753.17 & 51.54 & 1962.44 & -43.52 & 12.84 & 336.46 & 1578.95 & 366.44 & -680.00 & 3759.43 & 68.85 & 95.43 & 9.14 & 15.59 \\
\hline$\sigma 2 \mathrm{D}$ & 65.571 & 2.621 & 72.034 & -0.315 & -1.533 & 13.125 & 56.662 & 15.126 & -11.094 & 116.525 & 3.029 & 2.209 & -0.153 & 0.824 \\
\hline
\end{tabular}


Conti.....

\begin{tabular}{|c|c|c|c|c|c|c|c|c|c|c|c|c|c|c|}
\hline \multirow[t]{2}{*}{ Covariance } & \multicolumn{2}{|c|}{ Grain weight per spike } & \multicolumn{2}{|c|}{ Grain per spike } & \multicolumn{2}{|c|}{ Test weight } & \multicolumn{2}{|c|}{ Grain yield per plant } & \multicolumn{2}{|c|}{ Flag leaf area } & \multicolumn{2}{|c|}{ Harvest Index } & \multicolumn{2}{|c|}{ Biological Yield } \\
\hline & $\mathrm{S}$ & NS & $\mathrm{S}$ & NS & $\mathrm{S}$ & NS & $\mathrm{S}$ & NS & $\mathrm{S}$ & NS & $\mathrm{S}$ & NS & $\mathrm{S}$ & NS \\
\hline Cov HS (Line) & 0.000 & 0.000 & 13.276 & -4.208 & 5.371 & -2.480 & -1.323 & 0.853 & 20.790 & -14.743 & -39.604 & -1.025 & -5.759 & -8.601 \\
\hline Cov HS (Tester) & 0.065 & -0.010 & 21.54 & -5.613 & -2.694 & -0.524 & 1.039 & -1.166 & 27.801 & -5.025 & 33.464 & -6.912 & 12.063 & -9.410 \\
\hline Cov HS (Average) & -0.121 & -0.264 & -80.561 & -95.471 & -53.708 & -29.071 & -22.55 & -27.17 & -48.80 & -153.86 & -277.02 & -231.679 & -103.51 & -237.89 \\
\hline Cov FS & 0.258 & 0.274 & 171.405 & 99.219 & 85.803 & 34.991 & 39.779 & 42.982 & 125.142 & 222.064 & 399.530 & 291.425 & 198.144 & 378.420 \\
\hline Genetic components & & & & & & & & & & & & & & \\
\hline o2gca (Lines) & 0.000 & 0.000 & 13.276 & -4.208 & 5.371 & -2.480 & -1.323 & 0.853 & 20.790 & -14.743 & -39.604 & -1.025 & -5.759 & -8.60 \\
\hline$\sigma 2 \mathrm{gca}($ Testers $)$ & -0.001 & -0.010 & 53.10 & -16.83 & 21.48 & -9.92 & -5.29 & 3.41 & 83.16 & -58.97 & -158.41 & -4.10 & -23.03 & -34.40 \\
\hline б2gca (Parents) & 5.94 & -0.94 & 2040.54 & -536.07 & -212.92 & -62.52 & 86.60 & -100.98 & 2654.63 & -545.77 & 2807.57 & -635.14 & 1063.17 & -907.90 \\
\hline$\sigma 2 \mathrm{sca}$ & -0.24 & -0.52 & -161.12 & -190.94 & -107.41 & -58.14 & -45.10 & -54.34 & -97.59 & -307.73 & -554.04 & -463.35 & -207.02 & -475.79 \\
\hline$\sigma 2 \mathrm{gca} / \sigma 2 \mathrm{sca}$ & -24.59 & 1.79 & -12.665 & 2.807 & 1.982 & 1.075 & -1.920 & 1.85 & -27.19 & 1.77 & -5.06 & 1.371 & -5.13 & 1.90 \\
\hline$\sigma 2 \mathrm{~A}$ & 11.88 & -1.89 & 4081.09 & -1072.1 & -425.85 & -125.05 & 173.21 & -201.97 & 5309.26 & -1091.54 & 5615.15 & -1270.28 & 2126.35 & -1815.81 \\
\hline$\sigma 2 \mathrm{D}$ & 0.40 & -0.12 & 151.27 & -50.06 & -3.75 & -3.73 & 15.87 & 0.45 & 169.02 & 8.19 & 225.93 & -48.71 & 122.17 & 25.91 \\
\hline
\end{tabular}

Table.3 Estimates of general combining ability effects of lines and tester parent's in wheat

\begin{tabular}{|c|c|c|c|c|c|c|c|c|c|c|c|c|c|c|}
\hline Parents & \multicolumn{2}{|c|}{ Days to heading } & \multicolumn{2}{|c|}{ Days to flowering } & \multicolumn{2}{|c|}{ Days to maturity } & \multicolumn{2}{|c|}{ Grain filling period } & \multicolumn{2}{|c|}{ Plant height } & \multicolumn{2}{|c|}{ Tiller/plant } & \multicolumn{2}{|c|}{ Spike length } \\
\hline Lines & $\mathrm{S}$ & NS & $\mathrm{S}$ & NS & $\mathrm{S}$ & NS & $\mathrm{S}$ & NS & $\mathrm{S}$ & NS & $\mathrm{S}$ & NS & $\mathrm{S}$ & NS \\
\hline MACS 6222 & -1.976 & -1.738 & -1.810 & -1.333 & 0.452 & 0.667 & 2.262 & 2.000 & -1.322 & -6.445 & -0.249 & -0.346 & 0.512 & -0.151 \\
\hline GW 322 & -1.310 & 0.095 & -1.310 & 0.667 & 0.286 & 0.167 & 1.595 & -0.500 & 0.635 & -2.353 & -0.880 & 0.250 & $1.280^{* * *}$ & 0.179 \\
\hline GW 173 & 0.857 & 0.595 & 1.024 & 0.167 & 0.119 & -0.667 & -0.905 & -0.833 & -2.719 & 1.935 & 0.076 & 1.752 & 0.275 & 0.598 \\
\hline HI 1544 & 0.190 & -0.238 & 0.190 & -0.167 & -0.048 & 0.167 & -0.238 & 0.333 & -6.215 & -0.682 & -1.362 & -1.310 & -0.416 & -0.426 \\
\hline PBW 175 & 0.524 & -0.238 & 0.024 & -0.833 & -0.881 & -0.167 & -0.905 & 0.667 & 3.258 & 1.825 & 0.485 & 0.084 & 0.229 & 0.138 \\
\hline DL 803-3 & -0.143 & 0.762 & 0.190 & 0.667 & -0.548 & -0.667 & -0.738 & -1.333 & 0.268 & 0.492 & 0.596 & 0.669 & -0.875 & -0.166 \\
\hline C 306 & 1.857 & 0.762 & 1.690 & 0.833 & 0.619 & 0.500 & -1.071 & -0.333 & 6.095 & 5.228 & 1.333 & -1.100 & -1.005 & -0.173 \\
\hline SE (gca for line) & 1.230 & 0.884 & 1.566 & 0.796 & 0.938 & 0.822 & 1.620 & 1.099 & 2.934 & 3.280 & 0.908 & 1.457 & 0.536 & 0.414 \\
\hline Tester & & & & & & & & & & & & & & \\
\hline PBW 343 & $-3.643^{* * *}$ & 0.214 & $-3.857 * *$ & 0.119 & -0.357 & 1.548 ** & $3.500 * *$ & $1.429 * *$ & -1.116 & -1.603 & 0.695 & -0.460 & $-0.108 * *$ & $-0.031 * *$ \\
\hline HD 2932 & 1.571 & 0.714 & 1.500 & 0.405 & -0.071 & $-1.381^{* *}$ & -1.571 & $-1.786^{* *}$ & -0.523 & -3.595 & -0.998 & 1.263 & $-0.251 * *$ & $0.452 * *$ \\
\hline GW 190 & $2.071^{* * *}$ & -0.929 & 2.357 *** & -0.524 & 0.429 & -0.167 & -1.929 & 0.357 & 1.640 & $5.198 * *$ & 0.303 & -0.803 & $0.359 * *$ & $-0.421^{* * *}$ \\
\hline SE (gca for tester) & 0.805 & 0.579 & 1.025 & 0.521 & 0.614 & 0.538 & 1.061 & 0.719 & 1.921 & 2.147 & 0.594 & 0.954 & 0.351 & 0.271 \\
\hline
\end{tabular}

Conti...

\begin{tabular}{|c|c|c|c|c|c|c|c|c|c|c|c|c|c|c|}
\hline \multirow{2}{*}{$\begin{array}{ll} & \text { Parents } \\
\text { Lines } & \end{array}$} & \multicolumn{2}{|c|}{ Grain weight per spike } & \multicolumn{2}{|c|}{ Grain per spike } & \multicolumn{2}{|c|}{ Test weight } & \multicolumn{2}{|c|}{ Grain yield per plant } & \multicolumn{2}{|c|}{ Flag leaf area } & \multicolumn{2}{|c|}{ Harvest Index } & \multicolumn{2}{|c|}{ Biological Yield } \\
\hline & $\mathrm{S}$ & NS & $\mathrm{S}$ & NS & $\mathrm{S}$ & NS & $\mathrm{S}$ & NS & $\mathrm{S}$ & NS & $\mathrm{S}$ & NS & $\mathrm{S}$ & NS \\
\hline MACS 6222 & -0.151 & 0.085 & 1.078 & 2.467 & $-7.979 * *$ & 0.314 & -0.755 & 0.655 & 5.341 & 4.336 & 0.162 & -3.354 & -2.672 & 4.739 \\
\hline GW 322 & 0.118 & 0.256 & 6.080 & 0.859 & -0.832 & -0.361 & -0.198 & -0.003 & -1.154 & 0.368 & 1.297 & -2.364 & -1.487 & -2.526 \\
\hline GW 173 & 0.227 & 0.220 & -1.308 & 5.189 & 1.863 & 0.269 & $2.447 * *$ & 2.052 & 0.221 & 3.518 & 2.544 & 1.194 & -3.722 & 6.641 \\
\hline HI 1544 & 0.008 & -0.359 & $-7.422 * *$ & -0.308 & 1.498 & -2.915 & $-1.835^{* *}$ & -2.378 & -5.326 & -4.299 & 0.337 & -4.480 & -1.460 & -3.648 \\
\hline PBW 175 & 0.033 & -0.114 & 3.025 & -4.032 & 1.095 & 0.855 & -0.236 & -1.905 & -0.762 & -1.367 & 2.620 & -5.383 & 0.271 & -1.294 \\
\hline DL 803-3 & -0.158 & -0.062 & 4.522 & 0.302 & 0.568 & -0.300 & $2.460 * *$ & $3.715 * *$ & -6.686 & -3.567 & -3.540 & 1.962 & 3.438 & 4.779 \\
\hline C 306 & -0.077 & -0.027 & -5.975 & -4.477 & 3.788 & 2.139 & $-1.883^{* *}$ & -2.136 & 8.366 & 1.011 & -3.421 & $12.424 * *$ & $5.633^{* *}$ & $-8.691 * *$ \\
\hline SE (gca for line) & 0.197 & 0.249 & 3.644 & 4.416 & 1.979 & 2.206 & 0.903 & 1.287 & 4.082 & 3.345 & 6.996 & 6.223 & 2.162 & 3.009 \\
\hline Tester & & & & & & & & & & & & & & \\
\hline PBW 343 & -0.187 & 0.074 & -1.708 & 1.238 & -0.040 & 1.318 & -0.200 & 0.129 & -3.949 & 2.517 & -5.046 & -3.532 & 4.729 & 2.939 \\
\hline HD 2932 & -0.125 & -0.103 & -4.157 & -0.380 & -1.048 & -1.159 & $-1.814^{* *}$ & 0.809 & -2.452 & -2.360 & -3.321 & 2.295 & -0.725 & -0.419 \\
\hline GW 190 & $0.312 * *$ & 0.029 & $5.865^{* *}$ & -0.857 & 1.088 & -0.159 & $2.013^{* *}$ & -0.938 & $6.401 * *$ & -0.158 & 8.367 & 1.237 & -4.004 & -2.520 \\
\hline SE (gca for tester) & 0.129 & 0.163 & 2.386 & 2.891 & 1.295 & 1.444 & 0.591 & 0.842 & 2.672 & 2.190 & 4.580 & 4.074 & 1.415 & 1.970 \\
\hline
\end{tabular}

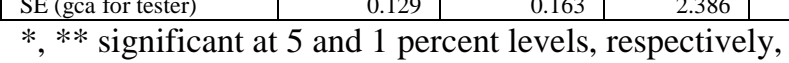




\section{Int.J.Curr.Microbiol.App.Sci (2017) 6(8): 2827-2834}

Table.4 Crosses showing significant specific combining ability (SCA) effect for grain yield and its attributes

\begin{tabular}{|c|c|c|c|c|c|c|c|c|c|c|c|c|c|c|}
\hline \multirow[t]{2}{*}{ Crosses } & \multicolumn{2}{|c|}{ Days to heading } & \multicolumn{2}{|c|}{$\begin{array}{c}\text { Days to } \\
\text { flowering }\end{array}$} & \multicolumn{2}{|c|}{$\begin{array}{l}\text { Days to } \\
\text { maturity }\end{array}$} & \multicolumn{2}{|c|}{$\begin{array}{c}\text { Grain filling } \\
\text { period }\end{array}$} & \multicolumn{2}{|c|}{ Plant height } & \multicolumn{2}{|c|}{ Tiller/plant } & \multicolumn{2}{|c|}{ Spike length } \\
\hline & $\mathrm{S}$ & NS & $\mathrm{S}$ & NS & $\mathrm{S}$ & NS & $\mathrm{S}$ & NS & $\mathrm{S}$ & NS & $\mathrm{S}$ & NS & $\mathrm{S}$ & NS \\
\hline GW 322 X GW 173 & -1.405 & 0.119 & -1.833 & -0.738 & -1.429 & 0.548 & 0.405 & 1.286 & -3.433 & -1.310 & 2.033 & 1.545 & -0.236 & 0.168 \\
\hline HI 1544 X PBW 343 & 2.429 & -1.381 & 3.333 & -1.238 & 0.738 & -0.619 & -2.595 & 0.619 & -3.470 & 1.652 & -0.674 & 1.544 & -1.366 & -0.525 \\
\hline MACS 6222 X PBW 343 & -1.571 & 0.262 & -2.024 & 0.190 & -0.762 & -0.333 & 1.262 & -0.524 & 2.277 & -0.401 & 2.941 & -1.476 & 0.875 & -0.542 \\
\hline HI 1544 X HD 2932 & -0.238 & 0.095 & -0.524 & 0.190 & 0.238 & -0.833 & 0.762 & -1.024 & -5.460 & 0.789 & 0.032 & 0.778 & -0.009 & -0.312 \\
\hline MACS 6222 X HD 2932 & -0.357 & -2.048 & 0.024 & -1.952 & 0.190 & -0.048 & 0.167 & 1.905 & 7.196 & -0.347 & 1.443 & 1.350 & 0.351 & -0.949 \\
\hline SE (sca) & 2.130 & 1.531 & 2.713 & 1.378 & 1.625 & 1.425 & 2.806 & 1.904 & 5.081 & 5.682 & 1.572 & 2.524 & 0.929 & 0.718 \\
\hline
\end{tabular}

Conti....

\begin{tabular}{|c|c|c|c|c|c|c|c|c|c|c|c|c|c|c|}
\hline \multirow[t]{2}{*}{ Crosses } & \multicolumn{2}{|c|}{ Grain weight per spike } & \multicolumn{2}{|c|}{ Grain per spike } & \multicolumn{2}{|c|}{ Test weight } & \multicolumn{2}{|c|}{ Grain yield per plant } & \multicolumn{2}{|c|}{ Flag leaf area } & \multicolumn{2}{|c|}{ Harvest Index } & \multicolumn{2}{|c|}{ Biological Yield } \\
\hline & $\mathrm{S}$ & NS & $\mathrm{S}$ & NS & $\mathrm{S}$ & NS & $\mathrm{S}$ & NS & $\mathrm{S}$ & NS & $\mathrm{S}$ & NS & $\mathrm{S}$ & NS \\
\hline GW 322 X GW 173 & 0.067 & -0.102 & 1.826 & -3.622 & 1.651 & 3.389 & $4.514 * *$ & 1.770 & -1.810 & 1.151 & 3.373 & 2.313 & 4.110 & 2.836 \\
\hline HI 1544 X PBW 343 & -0.207 & 0.255 & -7.286 & -0.286 & 0.171 & 3.379 & -4.091 & $6.845^{* *}$ & -2.795 & -3.584 & -4.009 & -8.005 & -0.030 & $18.919 * *$ \\
\hline MACS 6222 X PBW 343 & 0.111 & -0.107 & 6.191 & -2.974 & $0.750 * *$ & 1.499 & $5.417 * *$ & $-5.129 * *$ & -0.567 & -2.751 & -14.929 & 7.838 & $7.999 * *$ & $-11.230 * *$ \\
\hline HI 1544 X HD 2932 & -0.235 & -0.054 & -2.807 & -0.589 & -2.082 & 0.612 & -1.145 & $5.343 * *$ & -0.194 & -0.996 & 3.438 & 13.617 & $-8.165 * *$ & 2.580 \\
\hline MACS 6222 X HD 2932 & 0.355 & 0.434 & -2.915 & 2.982 & $1.514 * *$ & 1.650 & 4.071 & 2.991 & 2.649 & -4.176 & 9.676 & -5.247 & 3.771 & $13.757 * *$ \\
\hline SE (sca) & 0.341 & 0.431 & 6.312 & 7.649 & 3.428 & 3.822 & 1.564 & 2.229 & 7.070 & 5.793 & 12.117 & 10.778 & 3.744 & 5.212 \\
\hline
\end{tabular}

$*, * *$ significant at 5 and 1 percent levels, respectively, 
Significant negative GCA effects reveled that genotypes GW 190 and PBW 343 showed bad general combiner for spike length and C 306 for biological yield. However, HD 2932 was good general combiner for earliness to maturity and grain filling period as reflected by negative GCA effects. Rests of the parents were average combiner for grain yield having non-significant GCA effects. These results are in agreement with those of Singh et al., (1988) for grain per spike and biological yield; Yadav and Behl (2002) for days to flowering, tillers per plant, grain per spike, test weight and grain yield per plant.

Under stress environment, GW 190 were recorded good general combiner for grain yield per plant, spike length, grain weight per spike, grain per spike and flag leaf area; DL 803-3 for grain yield per plant and C 306 for biological yield under stress condition. Whereas PBW 343 were good general combiner for earliness as showing significant negative GCA effects for days to heading and days to flowering and bad general combiner for grain filling period. Significant negative GCA effects revealed that HD 2932 showed bad general combiner for grain yield per plant and spike length; C 306 for grain yield per plant and PBW 343 for spike length (Table 3). The similar reports were also published earlier by Drikvand et al., (2005) for days to heading, days to flowering, day to maturity, plant height, grain yield; Kamaluddin et al., (2007) for seeds per spike, tillers per plant and grain yield per plant under both water stress and non-stress conditions.

\section{Specific combining ability effects in under stress and non-stress condition}

Under non-stress condition, Significant positive SCA effects revealed that cross ' $\mathrm{HI}$ 1544 X HD 2932' and 'HI 1544 X PBW 343' having higher grain yield and also was good specific combiner for grain yield per plant;
'MACS 6222 X HD 2932' for biological yield. It was noticed that the crosses with high SCA had either one or both parents with average or good GCA effects. The similar results were also reported earlier by Khalifa $e t$ al., (1998) and Jatav et al., (2014) for grain yield per plant.

Under stress condition, significant positive SCA effects revealed that ' $\mathrm{GW} 322 \mathrm{X} \mathrm{GW}$ 173' and 'MACS $6222 \mathrm{X}$ PBW 343' were good specific combiner for grain yield per plant; 'MACS 6222 X HD 2932' for test weight. Whereas cross 'DL 803-3 X PBW 343 ' showed high mean grain yield but was average specific combiner for biological yield but it showed good specific combiner for yield. The superiority of average $X$ average or average $\mathrm{X}$ low combination may be due to the presence of genetic diversity among the parent and there could be some complementation indicating importance of non-additive effects. The similar results were also reported earlier by Srivastava et al., (2012) and Pansuriya et al., (2014) for grain yield per plant.

Analysis of combining ability in the present wheat material suggested an idea about breeding methodology to be applied and use of promising crosses for further improvement in wheat. In self-pollinated crops like wheat, SCA effects are not much important as they are mostly related to non-additive gene effects excluding those of arising from complementary gene action or linkage effects they cannot be fixed in pure lines. Further superiority of the hybrids might not indicate their ability to yield transgressive segregates; rather SCA would provide satisfactory criteria and expected to throw desirable transgressive segregates in later generations. Grain yield and major yield components revealed the significance of both additive and non-additive gene action for grain yield and its different components. The presence of both significant 
additive and non-additive genetic variances for grain yield and major yield attributing traits suggested that high performance of yield and contributing traits can be fixed in subsequent segregating generation of ' $\mathrm{HI}$ 1544 X HD 2932' and 'HI 1544 X PBW 343' in sown non-stress conditions and ' $\mathrm{GW} 322 \mathrm{X}$ GW 173' and 'MACS $6222 \mathrm{X}$ PBW' in sown stress conditions (Table 4). The good general combining parents DL 803-3, GW 190 and GW 173 may be used for varietal improvement through the recurrent selection, inter-mating and bi-parental mating in $F_{2}$ generation of promising crosses consisting for early sown stress situation. The parents DL 803-3 in early sowing, GW 322 and PBW 343 in late sowing identified as higher yielder under non-stress condition would be used for improvement for high yielding varieties through the simple / recurrent selection from segregating generations in wheat. DL 803-3 appeared to be promising under both water stress and non-stress condition may be used for varietal improvement through the recurrent selection, inter-mating and biparental mating in $\mathrm{F}_{2}$ generation. The significant specific cross ' $\mathrm{GW} 322$ X GW 173 ' having one promising parent for grain yield under stress condition would be advanced through simple / recurrent selection in segregating generations, which may lead in the fixation of both additive and non-additive components while making improvement in grain yield and its attributes.

\section{References}

Ali, Z., and Khan, A. S. 1998. Combining ability studies of some morphophysiological traits in bread wheat (Triticum aestivum L.) Pakistan Journal Agriculture Science, 35:1-3.

Chowdhry, M. A., Saeed, M. S.; Ihsan Khaliq and Muhammad Ahsan. 2005. Combining ability analysis for some polygenic traits in a $5 \times 5$ diallel cross of bread wheat (Triticum aestivum L.) Asian Journal of Plant Science, 4: (4) 405-408.

Dhadhal, B. A., and Dobariya, K. L. 2006. Combining ability analysis over

environments for grain yield and its components

in bread wheat (Triticum aestivum L.).N ational J.Plant Improv. 8 (2): 172-173.

Drikvand, R., Farshadfar, E. and Nazarian, F. 2005. Genetic study of some morphophysiological traits in bread wheat lines under dry land conditions using diallel crossing. Seed and Plant. 4 (20): 429444.

Jatav, M., Jatav S.K. and Kandalkar V.S. 2014. Combining ability and heterosis analysis of morpho- physiological characters in wheat. Annals of Plant and Soil Research 16(2): 79-83.

Kamaluddin, Rishi, M., Singh, Lal C. Prasad, Malik Z. Abdin and Arun K. Joshi. 2007. Combining ability analysis for grain filling duration and yield traits in spring wheat (Triticum aestivum L. em. Thell.) Genetics and Molecular Biology, 30 (2): 411-416.

Kemthrone O., 1957. An introduction to genetical statistics. John Wiley and sons, Inc, Newyork.

Khalifa, M. A., Ismail, A. A.; El-Nagar, G. R. and Amin, I. A. 1998. Genetical studies of earliness, grain yield and its components of bread wheat. Assiut J. Agric. Sci. 5 (29): 59-69.

Mahmood Naeem and Chowdhry M. A. 2000. Inheritance of flag leaf in bread wheat genotypes. Wheat Information Service, 90: 7-12.

Muralia, S., and Sastry, E. V. D. 2001. Combining ability for germination and seedling establishment characters in bread wheat (Triticum aestivum L.) under normal and saline environments. Indian Journal of Genetics, 61: (1) 6970. 
Pansuriya, A. G., Dhaduk, L. K., Vanpariya, L. G., Savaliya, J. J., Patel, M. B., and Mehta, D. R. 2014. Combining ability over environmnets for grain yield and its components in bread wheat (Triticum aestivum L.). AGRES - An International e-Journal, 1(3): 39-46.

Singh, G., Bhullar, G. S. and Gill, K. S. 1988. Inheritance of yield and its components in an intervarietal cross of bread wheat. Crop Improvement, 15: (2) 200-202.

Singh, I., Sameena, S. and Singh J. 2000. Inheritance of some quantitative traits in bread wheat (Triticum aestivum L. em. Thell).Annals Agriculture Research, 21: (1) 51-54.
Srivastava, M. K., Singh, D., Sharma, S. 2012. Combining ability and gene action for grain yield and its components in bread wheat [(Triticum aestivum) (L.) em. Thell] Electronic Journal of Plant Breeding, 3: (1) 606611.

Vanpariya, L. G., Chovatia, V. P. and Mehta, D. R. 2006. Combining ability studies in bread wheat (Triticum aestivum L.) National Journal Plant Improvement, 8: (2) $132-137$.

Yadav, R., and Behl, R. K. 2002. Genetics of morpho-physiological characters and grain yield in wheat. National Journals Plant Improvements, 4: (2) 26-29.

\section{How to cite this article:}

Sunil Kumar Jatav, B.R. Baraiya and Kandalkar, V.S. 2017. Combining Ability for Grain Yield and Its Components Different Environments in Wheat. Int.J.Curr.Microbiol.App.Sci. 6(8): 2827-2834. doi: https://doi.org/10.20546/ijcmas.2017.608.337 